\title{
The long(er) road to gender equality
}

\author{
The COVID-19 pandemic is proving a major setback for achieving gender equality. Post-pandemic recovery efforts \\ must focus on supporting diversity and bridging the gender gap.
}

ife has become harder for women over the past year, more so for women of color and those belonging to underrepresented groups and underserved communities. This is what several reports assessing the collateral societal damage incurred by the COVID-19 pandemic indicate, pointing to repercussions that might be felt for generations. Indeed, according to the Global Gender Gap Report 2021 published by the World Economic Forum last month, the pandemic has shifted the attainment of gender equality by a whole generation, from 99.5 years to 135.6 years, in just 12 months.

The reasons behind this alarming estimate are multifarious. First, as outlined in a recent publication produced by the United Nations group UN Women on the impact of the COVID-19 pandemic on gender equality, the pandemic has been detrimental to women's health and safety. For example, $70 \%$ of the healthcare and social-care workforce are women, which makes them more likely to be frontline healthcare workers exposed to the risk of infection. In addition, stay-at-home orders have exacerbated the already high threat of domestic abuse against women and its repercussions on their physical and mental health. Global maternal and perinatal outcomes have also worsened during this time, including an increase in maternal deaths and depression ${ }^{1}$.

The World Economic Forum report notes that women, who are already at a disadvantage compared with men when it comes to job security and earning power, have been disproportionately affected on the economic front. Being overrepresented in sectors that were hardest hit by lockdown mandates and the economic downturn, such as the consumer sector, more women than men are estimated to have lost their jobs during the pandemic. Moreover, women perform much more unpaid work than men do, by taking on a higher share of domestic work, caregiving and homeschooling responsibilities. Research for UN Women by the market-research firm Ipsos revealed that in most of the countries surveyed, the time women devoted to childcare every week increased to more than 30 hours, nearly a full work week, during the pandemic. Although men also took on more domestic and caregiving tasks, this was consistently less than it was for women, which raised stress and the domestic and professional pressures faced by the latter.

Women in science had to address similar time and multi-tasking challenges, often having to juggle increased responsibilities on the home front with conducting their lab work in shifts and for limited hours, and running a lab, mentoring trainees, and teaching or attending classes remotely. These pandemic-imposed complications have compounded the well-documented gender imbalances in the scientific world. According to UNESCO (United Nations Educational, Scientific and Cultural Organization), women represent only one third of the global researcher community. Although this imbalance is multifactorial, it reflects in great part the traditionally lower participation of women and girls in education and ultimately professions relating to science, technology, engineering and mathematics (STEM). In the World Economic Forum report, this trend is clear for countries at both ends of the gender-equality spectrum. For instance, in Iceland and Finland, the world's top two countries for gender equality, only $10-13 \%$ of women graduates opt for STEM disciplines, compared with more than double this proportion for male graduates; these numbers are similar to those for the USA, which ranks 30 th, and Indonesia, which is listed as 101st.

Whether the present economic crisis will spur more women and girls to elect to pursue STEM education and career tracks, given that these are more relevant to the current and developing job markets, remains to be seen. However, early reports suggest that the disruptions to work and productivity over the past year may be hampering the research output of women more than that of men. For example, women deposited fewer preprints and registered fewer registered reports, including clinical trials, on the basis of estimates early in the pandemic ${ }^{2}$. Although research on SARS-CoV2 and COVID-19 has been booming, fewer women than men were noted as first and last authors in relevant publications in the first half of 2020 (ref. ${ }^{3}$ ). Although suggestive, the shortcomings of the name-based gender-prediction methods used in these reports and the limited time period assessed mean that more time and deeper, more rigorous study are needed to reveal the true extent to which the pandemic may be stymying the research efforts of women.

More than a year since the COVID-19 crisis started and as vaccination efforts are ramping up across the globe, even if at different speeds and with variable efficiency, the world has started to look ahead to a post-pandemic reality. As plans are being formulated to support ailing economies and the return to a semblance of normal daily life, it is essential to address the widening gender gap using the lessons learned in the past 12 months. For instance, beyond the broader cultural shift that is needed to equalize the caregiving responsibilities of working parents, adopting policies of paid parental leave and provision or subsidizing of childcare facilities near the place of work are necessary to support a gender-balanced research enterprise in the future. Gender equality must become hardwired into hiring and funding processes to ensure retention and, ultimately, advancement to leadership positions of the best and the brightest. To that end, reassessing remuneration and acknowledging and closing pay gaps is essential. It is also important to recognize that rigid one-size-fits-all approaches to training and career structure can be detrimental not only to the career development of individual researchers but also to science as a whole. One lesson from 2020 is that building more flexibility and customization into training, work and funding processes and schedules can support talented people in conducting their work in efficient and innovative ways. More generally, adopting a gender-sensitive outlook across institutional and funder hierarchies will help redress gender imbalances and inequalities. Reverse-mentoring schemes could help those in leadership positions understand the cultural shifts, evolving needs and challenges faced by junior researchers, from trainees to group leaders. In the longer term, robust educational initiatives in formal and informal settings will be needed to remove societal gendered views about STEM disciplines and the position of women in the STEM labor market, aiming instead to nurture the proclivities of fledgling scientists from a young age regardless of gender. Such efforts would not only remedy gender-based biases and disparities but also foster diversity and inclusion more broadly. 
The COVID-19 pandemic has put gender inequalities in stark relief and has widened the already existing gender gap. As countries start to look beyond the pandemic, it is important to take the long view and make gender equality an integral part of recovery plans.

Published online: 26 April 2021

https://doi.org/10.1038/s43018-021-00206-8
References

7 1. Chmielewska, B. et al. Lancet Glob. Health https://doi, org/10.1016/S2214-109X(21)00079-6 (2021).

2. Viglione, G. Nature 581, 365-366 (2020).

3. Pinho-Gomes, Ana-C. et al. BMJ Glob. Health 5, e002922 (2020). 